\title{
Anisotropy in the Human Placenta in Pregnancies Complicated by Fetal Growth Restriction
}

\author{
Paddy J. Slator, Alison Ho, Spyros Bakalis, Laurence Jackson, \\ Lucy C. Chappell, Daniel C. Alexander, Joseph V. Hajnal, Mary Rutherford, \\ and Jana Hutter
}

\begin{abstract}
The placenta has a unique structure, which enables the transfer of oxygen and nutrients from the mother to the developing fetus. Abnormalities in placental structure are associated with major complications of pregnancy; for instance, changes in the complex branching structures of fetal villous trees are associated with fetal growth restriction. Diffusion MRI has the potential to measure such fine placental microstructural details. Here, we present in-vivo placental diffusion MRI scans from controls and pregnancies complicated by fetal growth restriction. We find that after 30 weeks' gestation fractional anisotropy is significantly higher in placentas associated with growth restricted pregnancies. This shows the potential of diffusion MRI derived measures of anisotropy for assessing placental function during pregnancy.
\end{abstract}

\section{Introduction}

Fetal growth restriction (FGR) is a condition where the developing fetus does not reach its full growth potential in-utero [13]. It constitutes a major pregnancy complication and is associated with a high degree of fetal mortality, morbidity and life-long complications [19]. Early onset FGR (defined as that diagnosed before 32 weeks' gestation) affects $0.5-1 \%$ of pregnancies and late onset FGR (diagnosed after 32

\section{P. J. Slator $(\bowtie) \cdot$ D. C. Alexander}

Centre for Medical Image Computing, Department of Computer Science, University College London, London, UK

e-mail: p.slator@ucl.ac.uk

A. Ho · S. Bakalis $\cdot$ L. C. Chappell

Women's Health Department, King's College London, London, UK

L. Jackson · J. V. Hajnal · M. Rutherford · J. Hutter

Centre for the Developing Brain, School of Biomedical Engineering and Imaging Sciences,

King's College London, London, UK

e-mail: jana.hutter@kcl.ac.uk

L. Jackson · J. V. Hajnal · J. Hutter

Biomedical Engineering Department, School of Biomedical Engineering and Imaging Sciences,

King's College London, London, UK

E. Özarslan et al. (eds.), Anisotropy Across Fields and Scales,

Mathematics and Visualization, https://doi.org/10.1007/978-3-030-56215-1_13 
weeks') affects 5-10\% of pregnancies [6, 9]. Early diagnosis and close monitoring are essential to optimize the outcome for pregnancies affected by FGR. Recently, pharmacological treatments have shown promise for severe early onset FGR cases [31]. However, while routine antenatal monitoring utilising symphysis-fundal height and ultrasound measurements can identify a significant proportion of FGR cases [10], detection remains a major problem-only $31 \%$ of cases were diagnosed during a previous study [11]. Detection is crucial as FGR is a leading cause of late unexpected stillbirth [11].

Post-delivery histopathological analysis shows a significant degree of characteristic pathologies in FGR placentas [15, 33], emphasising its importance and involvement in the cascade of events leading ultimately to sub-optimal growth. However, while post-delivery detection or confirmation plays an important role in increasing knowledge and possible causes of FGR, it comes too late to influence clinical care for these pregnancies. Therefore, recent novel developments, mainly using Magnetic Resonance Imaging (MRI) focus on studying the placenta during pregnancy to complement available antenatal screening.

\subsection{Placental Microstructure}

The placenta constitutes the key connection between mother and baby in utero and acts as the life support system for the growing fetus. Among its many functions are the exchange of oxygen and nutrients from the maternal blood circulation to the fetal blood, and the removal of waste products. The placenta comprises 10-40 individual lobules each constituting one key exchange unit. The transfer relies on a delicate and dynamically evolving microstructure within these units, focused around the fetal villi and depicted in Fig. 1A. These tree-like structures originate from the umbilical cord and contain fetal arteries and veins. They are bathed in maternal blood that enters the intervillous spaces from the spiral arteries at the level of the basal plate. A thin membrane called the syncytiotrophoblast separates maternal and fetal circulation, and allows the transport of oxygen and nutrients through it. Several histopathological features are associated with FGR, including elongated villous trees without the appropriate branching patterns [21] as illustrated schematically in Fig. 1B.

\subsection{Placental MRI}

Specific challenges of in-utero placental MRI include motion, such as maternal breathing and fetal movements, various air-tissue interfaces such as amniotic fluid, abdominal fat and bowel gas, and the suboptimal position of the imaging coil with respect to the organ of interest, especially for placentas located on the posterior wall of the uterus [5]. Another important limitation specifically for diffusion MRI arises 


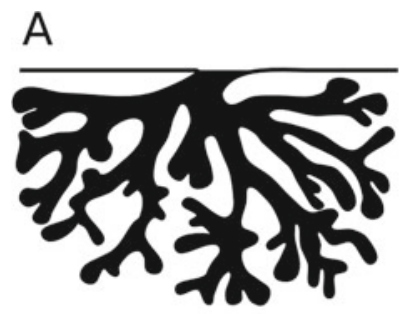

Healthy villous tree
(schematic)
B

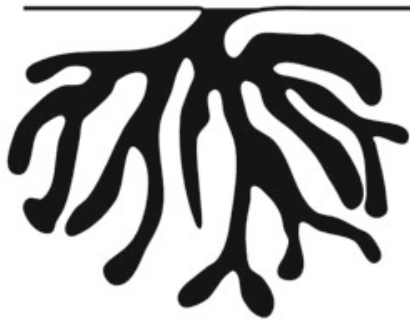

FGR villous tree (schematic)

Fig. 1 Schematic representation of single fetal villous trees in the placenta. Fetal blood flows through the convoluted branching structures, allowing nutrient exchange with the surrounding maternal blood. Panel A: complex branching structure of a healthy placenta. Panel B: pathological branching structure, associated with prengancy complications such as fetal growth restriction

from the safety requirement to reduce the acoustic sound level of the sequence to protect the fetal hearing. Therefore, typically the gradient slew rate is reduced, in consequence increasing the read-out length and thus the echo time resulting in lower Signal-to-Noise Ratios (SNR) [5, 16].

Diffusion MRI can reveal fine tissue microstructure details through sensitivity to the diffusion of water. By varying the strength and direction of diffusion gradients, the MRI sequence can be specifically tailored to microstructural features of interest. For example, sequences with a high number of distinct gradient directions can inform on tissue anisotropy and directionality. Such diffusion MRI sequences are commonly used to image white matter fibre tracts in the brain, however applications elsewhere in body exist, including in the placenta [3, 20, 27].

\section{Methods}

Pregnant women, recruited as part of a larger cohort for the Placental Imaging Project (PIP), underwent an MRI scan between 20 and 40 weeks of gestation. Informed consent was obtained (REC 14/LO/1169) and the scan was performed on a clinical 3T Philips Achieva (Best, The Netherlands) scanner using the 32-channel cardiac coil. All women were scanned in supine position under frequent monitoring of heart rate, saturation and blood pressure throughout the scan. Dedicated padding was provided to increase maternal comfort and verbal interaction was maintained.

After initial structural T2-weighted sequences of the entire uterus and the fetal brain in multiple orientations, a B0 map was acquired. An in-house developed tool for image based shimming was employed [12] to focus the shim on the placental parenchyma, avoiding air-tissue interfaces with bowel gas as much as possible. We 
next acquired a diffusion-prepared spin echo sequence. For some participants we used a modified sequence which acquires additional gradient-echos after the initial Spin Echo (e.g. [28]), although in this study we only utilised the first spin echo measurement. Conventional Stejskal-Tanner diffusion preparation was performed, with a total of $65 \mathrm{~b}$-value/b-vector combinations, optimized specifically for the placenta as previously described $[17,26]$, with three diffusion gradient directions at $b=[5$, $10,25,50,100,200,400,600,1200,1600] \mathrm{s} / \mathrm{mm}^{2}$, eight directions at $\mathrm{b}=18 \mathrm{~s} / \mathrm{mm}^{2}$, seven at $b=36 \mathrm{~s} / \mathrm{mm}^{2}$, fifteen at $b=800 \mathrm{~s} / \mathrm{mm}^{2}$, and six $b=0$ volumes. Further parameters include FOV $=[300-340] \times 320 \times 84 \mathrm{~mm}, \mathrm{TR}=7 \mathrm{~s}, \mathrm{SENSE}=2.5$, halfscan $=0.6$, resolution $=3 \mathrm{~mm}^{3}$ for scans with additional gradient-echos and $2 \mathrm{~mm}^{3}$ otherwise. The total acquisition time was $8 \mathrm{~min} 30 \mathrm{~s}$. Fat was suppressed with SPIR saturation pulses. The acquisition plane was coronal to the mother, chosen to assure that the in-plane direction coincides with the longest placental dimensions in mostly anterior and posterior placentas.

To correct for motion, the diffusion weighted volumes were registered non-rigidly to a common template using the ANTs multivariate template construction tool with the cross-correlation similarity metric [1]. Subsequently, a region of interest (ROI) comprising the whole placenta and adjacent basal placenta was manually segmented in all slices of the first $b=0$ volume. We estimated diffusion tensor, mean diffusivity (MD), and fractional anisotropy (FA) maps — using all diffusion weightings — for the motion corrected scans using MRTrix [32].

\subsection{Recruitment}

We include a total of twenty-nine participants in this study, who were categorised as follows. Sixteen women were normal uncomplicated control pregnancies; their outcomes were obtained and checked to ensure that no new diagnosis of pre-eclampsia, gestational hypertension, fetal growth restriction or gestational diabetes had occurred, and their birthweight was greater than the 5th centile (by INTERGROWTH-21st). Seven women were diagnosed with fetal growth restriction, detected from antenatal ultrasound assessments. We also include six women recruited as uncomplicated control pregnancies who gave birth to a baby under the 5th centile, but did not have a formal antenatal FGR diagnosis. Although these could simply be constitutionally small babies, they could also be undiagnosed FGR cases, so we hence analysed them as a separate cohort. Five of the six below the 5th centile had co-morbidities, such as chronic hypertension in pregnancy $(\mathrm{CHTN})$, or pre-eclampsia (PE). The patient population characteristics are given in Table 1. 
Table 1 Scan details for all participants. FGR denotes participants diagnosed with fetal growth restriction according to guidelines [13]. Under 5\% denotes participants with no FGR diagnosis who delivered a baby weighting under the 5 th percentile

\begin{tabular}{|c|c|c|}
\hline Participant ID & Cohort & Gestational age at scan \\
\hline 1 & Control & 23.86 \\
\hline 2 & Control & 26.14 \\
\hline 3 & Control & 26.72 \\
\hline 4 & Control & 26.72 \\
\hline 5 & Control & 27.14 \\
\hline 6 & Control & 27.14 \\
\hline 7 & Control & 28.29 \\
\hline 8 & Control & 28.86 \\
\hline 9 & Control & 28.86 \\
\hline 10 & Control & 29.67 \\
\hline 11 & Control & 29.86 \\
\hline 12 & Control & 31.29 \\
\hline 13 & Control & 33.43 \\
\hline 14 & Control & 35.57 \\
\hline 15 & Control & 36.29 \\
\hline 16 & Control & 36.43 \\
\hline 21 & FGR & 22.0 \\
\hline 22 & FGR & 23.42 \\
\hline 23 & FGR & 28.57 \\
\hline 24 & FGR & 29.57 \\
\hline 25 & FGR & 30.85 \\
\hline 26 & FGR & 32.85 \\
\hline $28($ Scan 1$)$ & FGR + CHTN & 30.71 \\
\hline $28($ Scan 2) & FGR + PE & 34.14 \\
\hline 29 & Under $5 \%$ & 21.29 \\
\hline 30 & Under $5 \%$ & 25.72 \\
\hline 31 & Under 5\% + CHTN & 38.0 \\
\hline 32 & Under 5\% + CHTN & 19.86 \\
\hline 33 & Under 5\% + PE & 28.71 \\
\hline $34($ Scan 1) & Under 5\% + PE & 31.42 \\
\hline 34 (Scan 2) & Under 5\% + PE & 33.42 \\
\hline
\end{tabular}

\section{Results}

Figures 2 and 3 display FA maps, ordered by gestational age (GA) at scanning time, for control and growth restricted participants respectively. We next examine the evolution of FA values over gestation (Fig. 4). Finally, we compare FA values 


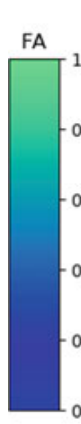

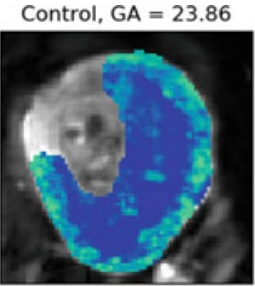

Control, GA $=26.72$

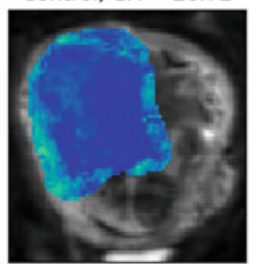

Control, GA $=28.29$

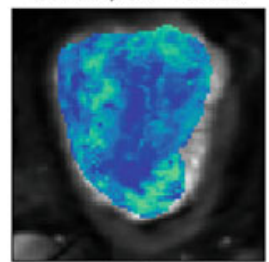

Control, GA $=29.67$

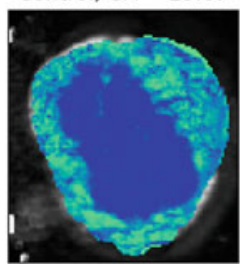

Control, GA = 33.43

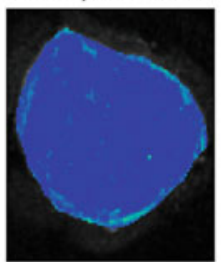

Control, GA $=36.43$

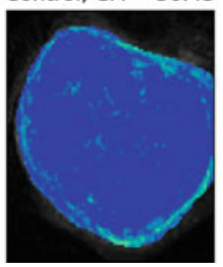

Control, GA $=26.14$

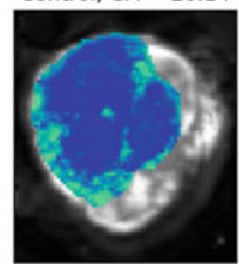

Control, GA $=27.14$

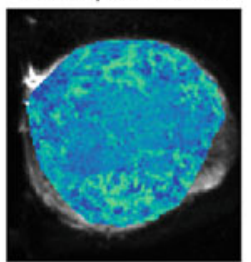

Control, GA $=28.86$

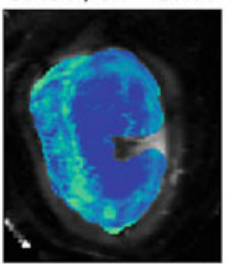

Control, GA $=29.86$
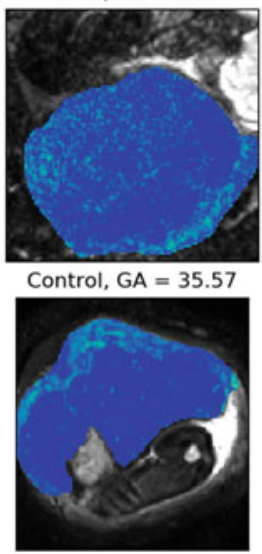

Control, GA $=26.72$

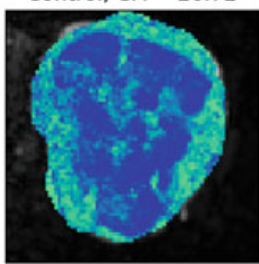

Control, GA $=27.14$

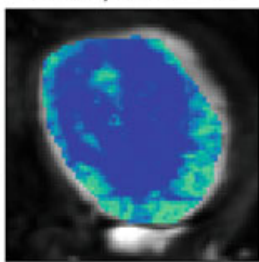

Control, GA $=28.86$

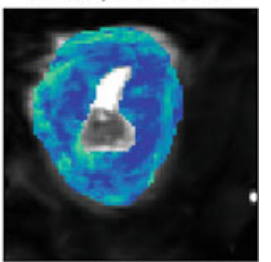

Control, GA $=31.29$

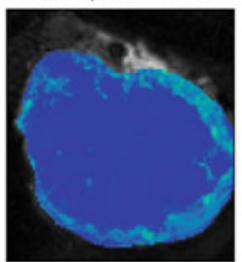

Control, GA = 36.29

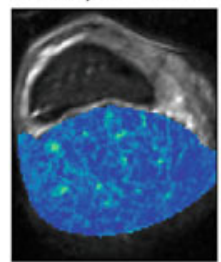

Fig. 2 Fractional anisotropy maps for all control participants. The boundary between the uterine wall (higher FA) and placenta (lower FA) is clear in most placentas with gestational age less than 30 weeks 

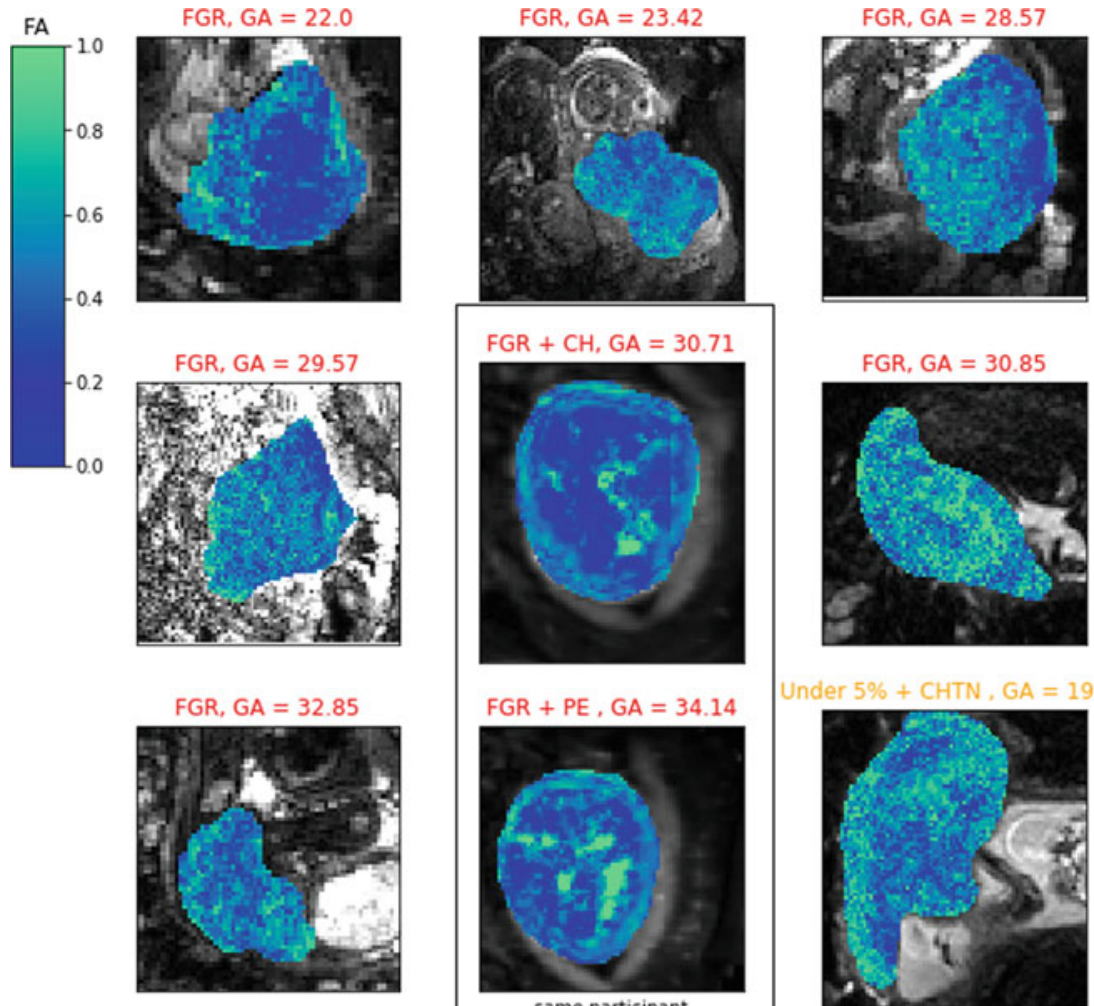

same participant
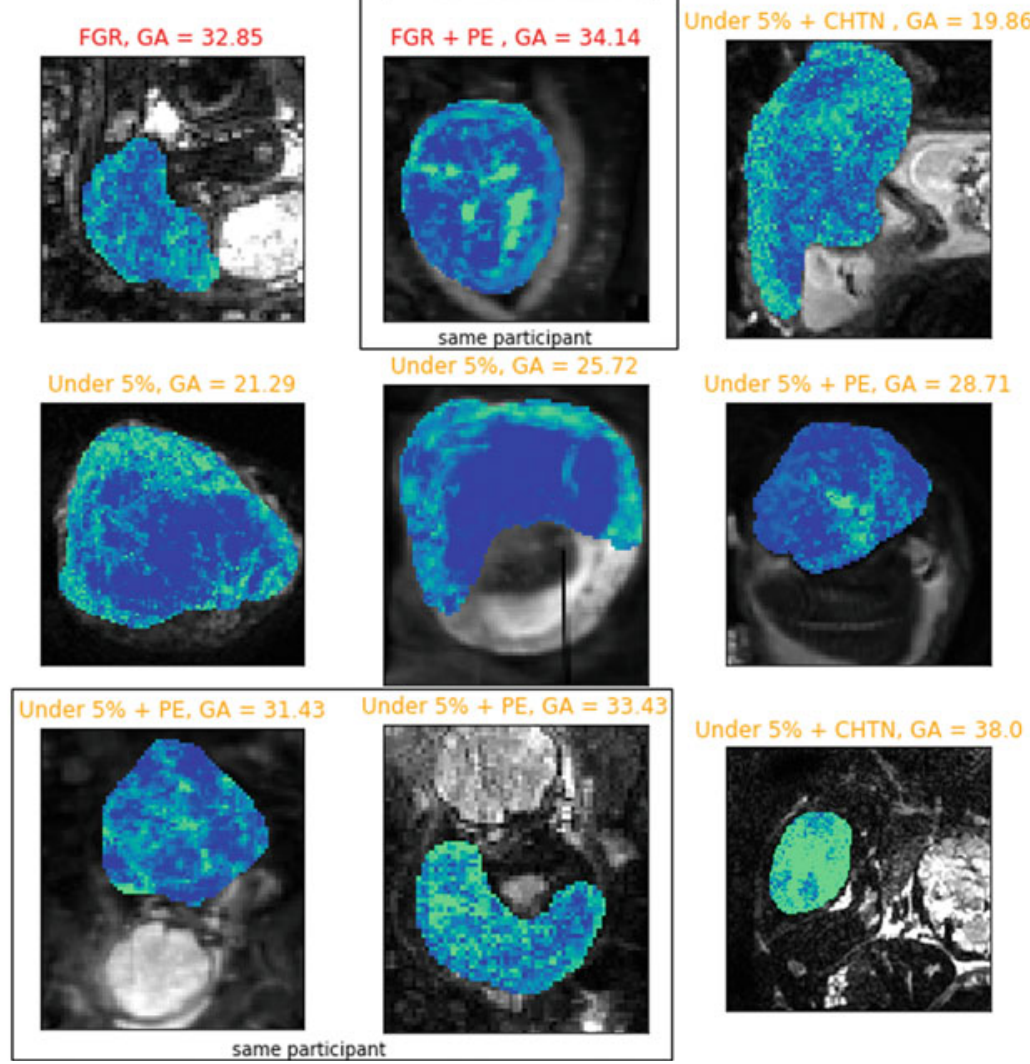

Fig. 3 Fractional anisotropy maps for FGR and low birthweight cohorts 


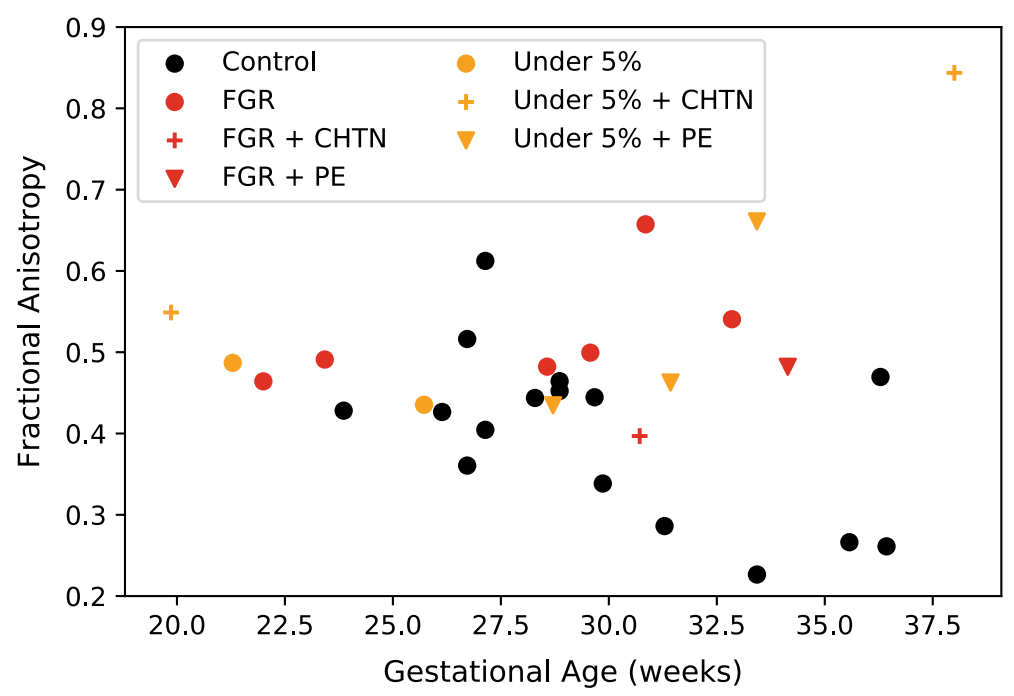

Fig. 4 Mean FA across placenta and uterine wall as a function of gestational age

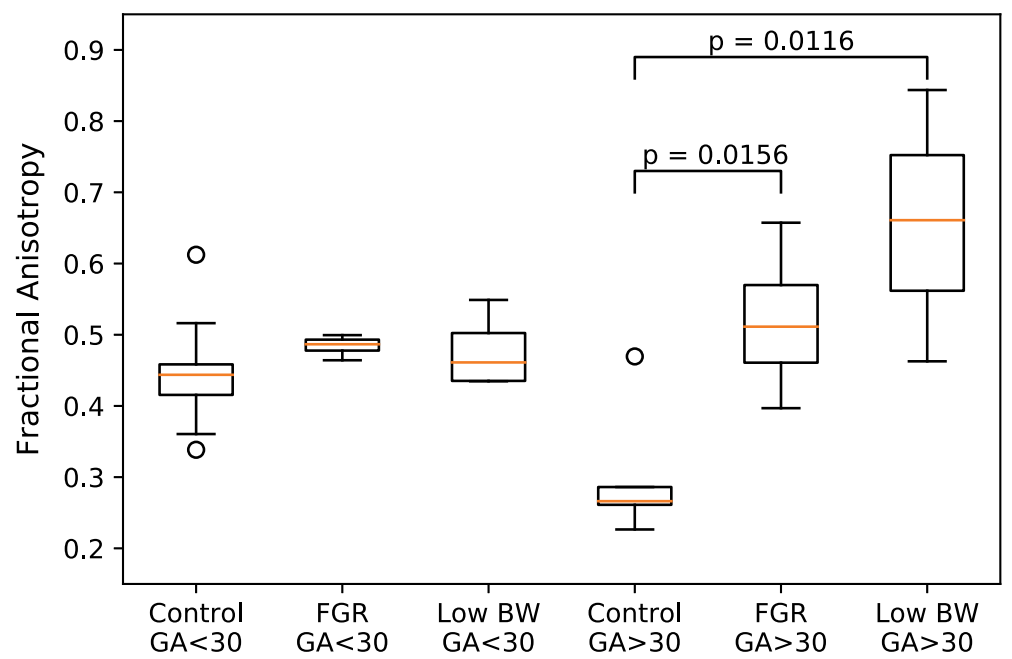

Fig. 5 Mean FA across placenta and uterine wall for the three cohorts, split at 30 weeks gestational age

between control and growth-restricted pregnancies (Fig. 5), assessing the potential of FA to inform on FGR-associated placental abnormalities.

The FA maps for all control participants (Fig. 2) show distinctive patterns in agreement with the literature [27] — the FA is lower in the placenta, and higher in 
the adjacent uterine wall. These observations likely represent isotropic nature of maternal blood pools and fetal villous tree branching within the placenta, and the prevalence of anisotropic fibrous muscle tissue in the uterine wall. Another factor in the high FA in the uterine wall may be the coherent orientation of vasculature in these areas. On the other hand, the distinction between the uterine wall and placenta is less clear in the growth restriction placentas (Fig. 3), and many of these maps show areas of high FA within the placenta.

Figure 4 plots the mean FA value within the ROI comprising the placenta and uterine wall. Control scans (Fig. 4, black dots) show an apparent decrease in mean FA over gestation, potentially reflecting microstructural changes during normal placenta maturation. On the other hand, growth restriction scans (Fig. 4, red and yellow dots), do not show a clear trend over gestation. At early gestational ages, control and compromised placentas have comparable mean FA values. Due to the downward FA trend in control placentas, from around 30 weeks gestation growth restriction placentas appear to have considerably lower FA values than control placentas. This difference was statisically significant, both when comparing controls with the combined FGR and low birth weight cohorts ( $p=0.005$, independent samples t-test), and when comparing controls to these two cohorts separately (Fig. 5). We tested if trends over GA differ between controls and growth restriction (i.e. FGR and under $5 \%$ ) cohorts by calculating a linear regression to predict mean FA based on GA, cohort, and the interaction between GA and cohort. The coefficients and p-values are given in Table 2, and Fig. 6 visualises the fits. The coefficient of the interaction term between GA and low birth weight is statistically significant $(p=0.002)$, suggesting a different trend over gestation.

Table 2 Coefficients and corresponding statistics from linear regression to predict mean FA based on GA, cohort, and GA $\times$ cohort (interaction term)

\begin{tabular}{l|l|l|l|l}
\hline Coefficient & Value & Standard error & $t$ & $P>|t|$ \\
\hline Intercept & 0.8457 & 0.193 & 4.384 & 0.000 \\
\hline Cohort (FGR) & -0.4541 & 0.313 & -1.449 & 0.160 \\
\hline Cohort (Under5) & -0.7159 & 0.259 & -2.765 & 0.011 \\
\hline GA & -0.0150 & 0.006 & -2.328 & 0.028 \\
\hline $\begin{array}{l}\text { GA } \times \text { Cohort } \\
(\text { FGR })\end{array}$ & 0.0188 & 0.011 & 1.769 & 0.089 \\
\hline $\begin{array}{l}\text { GA } \times \text { Cohort } \\
\text { (Under5) }\end{array}$ & 0.0299 & 0.009 & 3.412 & 0.002 \\
\hline
\end{tabular}




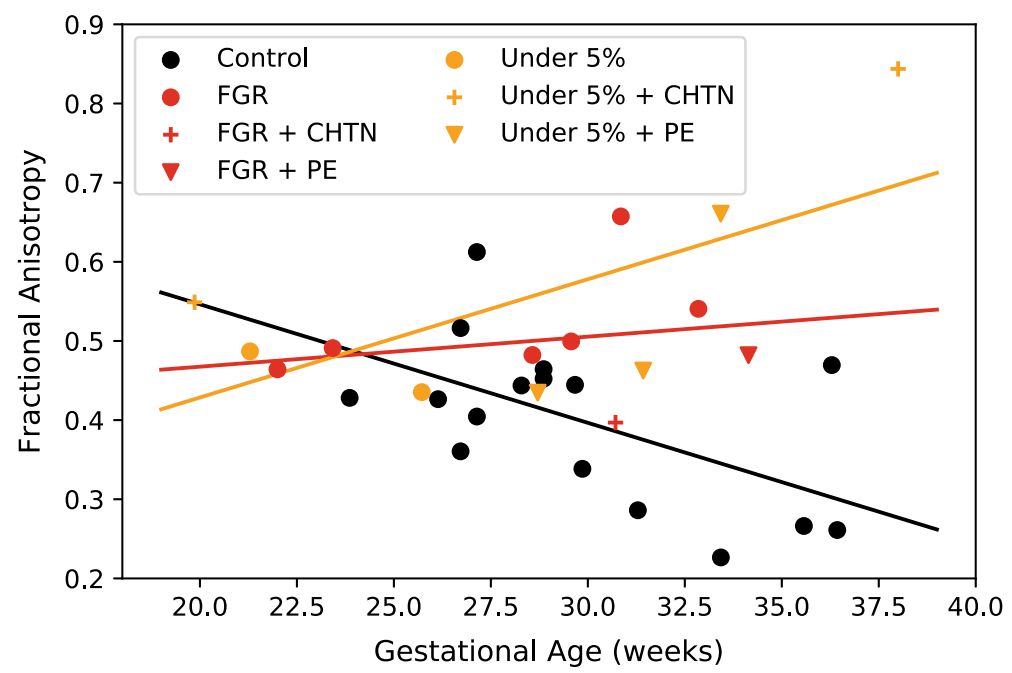

Fig. 6 Linear regression to predict mean FA based on GA, cohort, and GA $\times$ cohort

\section{Discussion and Conclusion}

This study visualizes and quantifies fractional anisotropy in placentas of both control pregnancies and those affected by growth restriction. Encouragingly we find that the FA is significantly different across gestation in growth restricted pregnancies compared to controls. The fractional anisotropy is a coarse measurement that averages over a number of tissue properties. There are hence a number of microstructural or functional changes that could explain this observation. Our initial speculations of plausible factors behind the changes in FA are as follows.

We observed a clear pattern of decreasing FA with gestational age for control placentas, suggesting that normal placental development causes a reduction in coherently orientated tissue. A consistent hypothesis is that this reduction in FA reflects the normal onset of terminal villi formation along the surfaces of intermediate villi, which occurs predominately during the third trimester [2]. On the other hand, we observed higher FA for placentas associated with growth restricted pregnancies after 30 gestational weeks. This is consistent with histological findings that FGR placentas show a lack of side-branching terminal villi [2].

In this study, we utilised an MR protocol with multiple b-values and gradient directions. However, we only fit a relatively simple model to the data, the diffusion tensor. This data can support more complex models, such as anisotropic IVIM-type models, which separately consider the perfusion (i.e. low b-value) and diffusion (high b-value) signal components (e.g. [26]), and may help disentangle the tissue microstructure changes underlying the observed difference in FA. Another potential approach is to use orientation distribution functions, which might reveal differences in complexity of the orientation of villi (e.g. [23]). 
There are a number of MRI-derived biomarkers that show promise for detecting placental dysfunction. These include: T2* relaxometry [18, 24, 25], which relates to oxygenation levels; structural measures [7]; apparent diffusion coefficient (ADC) [4, 14, 30], relating to global tissue structure; and intravoxel incoherent motion (IVIM) MRI perfusion fraction [8, 22, 29], which relates to blood flow. Thus far, dMRI-derived potential biomarkers (ADC and perfusion fraction) have considered tissue to be isotropic. Our results strongly suggest that the FA is also sensitive to placental dysfunction, and hence that quantifying tissue anisotropy is an additional important avenue for assessing placental health. It may be the case that multiple biomarkers need to be combined in order to best assess the health of an individual placenta. The fact that we see higher FA values for placentas with birth weight under the 5th percentile, as well as those diagnosed with FGR is interesting and merits further investigation. It is likely that a significant proportion of cases under the 5th percentile are undiagnosed FGR. Our results suggest that quantifying tissue anisotropy in the placenta could have a role to play in the detection of FGR, We will investigate this by combining further scanning with post-delivery placental histology to test the ability to distinguish FGR cases from small but otherwise healthy babies.

Acknowledgments We thank all mothers, midwives, obstetricians, and radiographers who played a key role in obtaining the datasets. This work was supported by the NIH Human Placenta Project grant 1U01HD087202-01 (Placenta Imaging Project [PiP]); Wellcome Trust (201374/Z/16/Z); EPSRC (N018702, M020533, EP/N018702/1); NIHR (RP-2014-05-019); the Wellcome EPSRC Centre for Medical Engineering at Kings College London (WT 203148/Z/16/Z) and by the National Institute for Health Research (NIHR) Biomedical Research Centre based at Guy's and St Thomas' NHS Foundation Trust and Kings' College London. The views expressed are those of the authors and not necessarily those of the NHS, the NIHR or the Department of Health.

\section{References}

1. Avants, B.B., Tustison, N.J., Song, G., Cook, P.A., Klein, A., Gee, J.C.: A reproducible evaluation of ANTs similarity metric performance in brain image registration. NeuroImage 54(3), 2033-2044 (2011). https://doi.org/10.1016/j.neuroimage.2010.09.025

2. Benirschke, K., Burton, G.J., Baergen, R.N.: Pathology of the Human Placenta. Springer, Heidelberg (2012). https://doi.org/10.1007/978-3-642-23941-0

3. Bonel, H.M., Stolz, B., Diedrichsen, L., Frei, K., Saar, B., Tutschek, B., Raio, L., Surbek, D., Srivastav, S., Nelle, M., Slotboom, J., Wiest, R.: Diffusion-weighted MR imaging of the placenta in fetuses with placental insufficiency. Radiology 257(3), 810-9 (2010). https://doi. org/10.1148/radiol.10092283

4. Bonel, H.M., Stolz, B., Diedrichsen, L., Frei, K., Saar, B., Tutschek, B., Raio, L., Surbek, D., Srivastav, S., Nelle, M., Slotboom, J., Wiest, R.: Diffusion-weighted MR Imaging of the Placenta in Fetuses with Placental Insufficiency. Radiology 257(3), 810-819 (2010). https:// doi.org/10.1148/radiol.10092283

5. Christiaens, D., Slator, P.J., Cordero-Grande, L., Price, A.N., Deprez, M., Alexander, D.C., Rutherford, M., Hajnal, J.V., Hutter, J., : In Utero Diffusion MRI. Top. Magn. Reson. Imaging 28(5), 255-264 (2019). https://doi.org/10.1097/RMR.0000000000000211

6. Crovetto, F., Triunfo, S., Crispi, F., Rodriguez-Sureda, V., Roma, E., Dominguez, C., Gratacos, E., Figueras, F.: First-trimester screening with specific algorithms for early- and late-onset 
fetal growth restriction. Ultrasound Obstet. Gynecol. 48(3), 340-348 (2016). https://doi.org/ 10.1002/uog. 15879

7. Damodaram, M., Story, L., Eixarch, E., Patel, A., McGuinness, A., Allsop, J., Wyatt-Ashmead, J., Kumar, S., Rutherford, M.: Placental MRI in intrauterine fetal growth restriction. Placenta 31(6), 491-8 (2010). https://doi.org/10.1016/j.placenta.2010.03.001

8. Derwig, I., Lythgoe, D., Barker, G., Poon, L., Gowland, P., Yeung, R., Zelaya, F., Nicolaides, K.: Association of placental perfusion, as assessed by magnetic resonance imaging and uterine artery Doppler ultrasound, and its relationship to pregnancy outcome. Placenta 34(10), 885-891 (2013). https://doi.org/10.1016/j.placenta.2013.07.006

9. Figueras, F., Caradeux, J., Crispi, F., Eixarch, E., Peguero, A., Gratacos, E.: Diagnosis and surveillance of late-onset fetal growth restriction. Am. J. Obstet. Gynecol. 218(2), S790S802.e1 (2018). https://doi.org/10.1016/j.ajog.2017.12.003

10. Gardosi, J., Kady, S.M., McGeown, P., Francis, A., Tonks, A.: Classification of stillbirth by relevant condition at death $(\mathrm{ReCoDe})$ : population based cohort study. BMJ (Clinical research ed.) 331(7525), 1113-7 (2005). https://doi.org/10.1136/bmj.38629.587639.7C

11. Gardosi, J., Madurasinghe, V., Williams, M., Malik, A., Francis, A.: Maternal and Fetal Risk Factors for Stillbirth: population based study. BMJ: Br. Med. J. 346, f108 (2013). https://doi. org/10.1136/bmj.f108

12. Gaspar, A.S., Nunes, R.G., Ferrazzi, G., Hughes, E.J., Hutter, J., Malik, S.J., McCabe, L., Baruteau, K.P., Rutherford, M.A., Hajnal, J.V., Price, A.N.: Optimizing maternal fat suppression with constrained image-based shimming in fetal MR. Magn. Reson. Med. (2018). https://doi. org/10.1002/mrm. 27375

13. Gordijn, S.J., Beune, I.M., Thilaganathan, B., Papageorghiou, A., Baschat, A.A., Baker, P.N., Silver, R.M., Wynia, K., Ganzevoort, W.: Consensus definition of fetal growth restriction: a Delphi procedure. Ultrasound Obstet. Gynecol. 48(3), 333-339 (2016). https://doi.org/10. 1002/uog. 15884

14. Gorkem, S.B., Coskun, A., Eslik, M., Kutuk, M.S., Ozturk, A.: Diffusion-weighted imaging of placenta in intrauterine growth restriction with worsening Doppler US findings. Diagn. Interv. Radiol. 25(4), 280-284 (2019). https://doi.org/10.5152/dir.2019.18358

15. Haeussner, E., Schmitz, C., Frank, H.G., Edler von Koch, F.: Novel 3D light microscopic analysis of IUGR placentas points to a morphological correlate of compensated ischemic placental disease in humans. Sci. Rep. 6(April), 24004 (2016). https://doi.org/10.1038/srep24004

16. Hutter, J., Harteveld, A.A., Jackson, L.H., Franklin, S., Bos, C., van Osch, M.J.P., O’Muircheartaigh, J., Ho, A., Chappell, L., Hajnal, J.V., Rutherford, M., De Vita, E.: Perfusion and apparent oxygenation in the human placenta (PERFOX). Magn. Reson. Med. 83(2), 549-560 (2019). https://doi.org/10.1002/mrm.27950

17. Hutter, J., Slator, P.J., Jackson, L., Gomes, A.D.S., Ho, A., Story, L., O’Muircheartaigh, J., Teixeira, R.P., Chappell, L.C., Alexander, D.C., Rutherford, M.A., Hajnal, J.V.: Multi-modal functional MRI to explore placental function over gestation. Magn. Reson. Med. 81(2), 11911204 (2019). https://doi.org/10.1002/mrm.27447

18. Ingram, E., Morris, D., Naish, J., Myers, J., Johnstone, E.: MR Imaging Measurements of Altered Placental Oxygenation in Pregnancies Complicated by Fetal Growth Restriction. Radiology 285(3), 953-960 (2017). https://doi.org/10.1148/radiol.2017162385

19. Malhotra, A., Allison, B.J., Castillo-Melendez, M., Jenkin, G., Polglase, G.R., Miller, S.L.: Neonatal Morbidities of Fetal Growth Restriction: Pathophysiology and Impact. Front. Endocrinol. 10, (2019). https://doi.org/10.3389/fendo.2019.00055

20. Melbourne, A., Aughwane, R., Sokolska, M., Owen, D., Kendall, G., Flouri, D., Bainbridge, A., Atkinson, D., Deprest, J., Vercauteren, T., David, A., Ourselin, S.: Separating fetal and maternal placenta circulations using multiparametric MRI. Magn. Reson. Med. 81(1), 350-361 (2018). https://doi.org/10.1002/mrm.27406

21. Mifsud, W., Sebire, N.J.: Placental pathology in early-onset and late-onset fetal growth restriction. Fetal Diagn. Therapy 36(2), 117-28 (2014). https://doi.org/10.1159/000359969

22. Moore, R., Strachan, B., Tyler, D., Duncan, K., Baker, P., Worthington, B., Johnson, I., Gowland, P.: In utero Perfusing Fraction Maps in Normal and Growth Restricted Pregnancy Measured 
Using IVIM Echo-Planar MRI. Placenta 21(7), 726-732 (2000). https://doi.org/10.1053/plac. 2000.0567

23. Seunarine, K.K., Alexander, D.C.: Multiple fibers. Beyond the diffusion tensor. In: Diffusion MRI: From Quantitative Measurement to In vivo Neuroanatomy, 2nd edn., pp. 105-123. Elsevier (2013). https://doi.org/10.1016/B978-0-12-396460-1.00006-8

24. Sinding, M., Peters, D.A., Frøkjaer, J.B., Christiansen, O.B., Petersen, A., Uldbjerg, N., Sørensen, A.: Placental magnetic resonance imaging T2* measurements in normal pregnancies and in those complicated by fetal growth restriction. Ultrasound Obstet. Gynecol. 47(6), 748-754 (2016). https://doi.org/10.1002/uog.14917

25. Sinding, M., Peters, D.A., Poulsen, S.S., Frøkjær, J.B., Christiansen, O.B., Petersen, A., Uldbjerg, N., Sørensen, A.: Placental baseline conditions modulate the hyperoxic BOLD-MRI response. Placenta 61, 17-23 (2018). https://doi.org/10.1016/J.PLACENTA.2017.11.002

26. Slator, P.J., Hutter, J., Ianuş, A., Panagiotaki, E., Rutherford, M., Hajnal, J.V., Alexander, D.C.: A framework for calculating time-efficient diffusion MRI protocols for anisotropic IVIM and an application in the placenta. In: Bonet-Carne, E., Grussu, F., Ning, L., Sepehrband, F., Tax, C. (eds.) Computational Diffusion MRI. Springer, Berlin (2019)

27. Slator, P.J., Hutter, J., McCabe, L., Gomes, A.D.S., Price, A.N., Panagiotaki, E., Rutherford, M.A., Hajnal, J.V., Alexander, D.C.: Placenta microstructure and microcirculation imaging with diffusion MRI. Magn. Reson. Med. 80(2), 756-766 (2018). https://doi.org/10.1002/mrm. 27036

28. Slator, P.J., Hutter, J., Palombo, M., Jackson, L.H., Ho, A., Panagiotaki, E., Chappell, L.C., Rutherford, M.A., Hajnal, J.V., Alexander, D.C.: Combined diffusion-relaxometry MRI to identify dysfunction in the human placenta. Magn. Reson. Med. 82(1), 95-106 (2019). https:// doi.org/10.1002/mrm.27733

29. Sohlberg, S., Mulic-Lutvica, A., Olovsson, M., Weis, J., Axelsson, O., Wikström, J., Wikström, A.K.: Magnetic resonance imaging-estimated placental perfusion in fetal growth assessment. Ultrasound Obstet. Gynecol. 46(6), 700-705 (2015). https://doi.org/10.1002/uog.14786

30. Song, F., Wu, W., Qian, Z., Zhang, G., Cheng, Y.: Assessment of the Placenta in Intrauterine Growth Restriction by Diffusion-Weighted Imaging and Proton Magnetic Resonance Spectroscopy. Reprod. Sci. 24(4), 575-581 (2017). https://doi.org/10.1177/1933719116667219

31. Spencer, R., Ambler, G., Brodszki, J., Diemert, A., Figueras, F., Gratacós, E., Hansson, S.R., Hecher, K., Huertas-Ceballos, A., Marlow, N., Marsál, K., Morsing, E., Peebles, D., Rossi, C., Sebire, N.J., Timms, J.F., David, A.L.: EVERREST Consortium: EVERREST prospective study: a 6-year prospective study to define the clinical and biological characteristics of pregnancies affected by severe early onset fetal growth restriction. BMC Pregnancy Childbirth 17(1), 43 (2017). https://doi.org/10.1186/s12884-017-1226-7

32. Tournier, J.D., Smith, R., Raffelt, D., Tabbara, R., Dhollander, T., Pietsch, M., Christiaens, D., Jeurissen, B., Yeh, C.H., Connelly, A.: MRtrix3: a fast, flexible and open software framework for medical image processing and visualisation. NeuroImage 202, 116137 (2019). https://doi. org/10.1016/j.neuroimage.2019.116137

33. Vedmedovska, N., Rezeberga, D., Teibe, U., Melderis, I., Donders, G.G.G.: Placental pathology in fetal growth restriction. Eur. J. Obstet. Gynecol. Reprod. Biol. 155(1), 36-40 (2011). https:// doi.org/10.1016/j.ejogrb.2010.11.017 
Open Access This chapter is licensed under the terms of the Creative Commons Attribution 4.0 International License (http://creativecommons.org/licenses/by/4.0/), which permits use, sharing, adaptation, distribution and reproduction in any medium or format, as long as you give appropriate credit to the original author(s) and the source, provide a link to the Creative Commons licence and indicate if changes were made.

The images or other third party material in this chapter are included in the chapter's Creative Commons licence, unless indicated otherwise in a credit line to the material. If material is not included in the chapter's Creative Commons licence and your intended use is not permitted by statutory regulation or exceeds the permitted use, you will need to obtain permission directly from the copyright holder. 